\title{
FAST ION INSTABILITY IN REAL LATTICE
}

\author{
G. V. Stupakov \\ Stanford Linear Accelerator Center, Stanford University, P.O. Box 4349, Stanford, CA 94309
}

\begin{abstract}
The ionization of residual gas by an electron beam in an accelerator generates ions that can resonantly couple to the beam through a wave propagating in the beam-ion system. The original theory of the Fast Ion Instability $[1,2]$ was developed assuming both a constant external focusing and the beam size. The theory predicts an instability in which an initial perturbation grows as $\sim \exp (\alpha \sqrt{t})$. In the present paper we consider a more realistic model that takes into account variation of the beta function in the lattice and associated with it variation of the beam size. We find that, in combination with ion decoherence effect, the spatial inhomogeneity can result in 1) purely exponential growth, $\sim \exp (\Gamma t)$, and 2) typically smaller growth rates. Detailed calculations are performed for the lattice of the Advanced Light Source at the LBL.
\end{abstract}

\section{INTRODUCTION}

A fast beam-ion instability which is caused by the interaction of a single electron bunch train with the residual gas ions $[1,2]$ can be of potential danger in future high-current, low-emittance accelerators. The instability mechanism is the same in both linacs and storage rings assuming that the ions are not trapped from turn-to-turn. The ions generated by the head of the bunch train oscillate in the transverse direction and resonantly interact with the betatron oscillations of the subsequent bunches, causing the growth of the initial perturbation of the beam. First experimental observation of the fast ion instability on the Advanced Light Source at the LBL has been recently reported in [3].

The original model of the instability developed in Ref. $[1,2]$ neglected the spatial variation of the beta function and ion frequency along the beam path. Evidently, this assumption somewhat overestimates the growth rate of the instability because it exaggerates the synchronism between the oscillations of ions located in different positions. It turns out that inclusion of the effect of the ion frequency variation indeed weakens the instability [4]. This effect is especially important for TBA and Chasman-Green lattices used in some synchrotron light sources and characterized by large excursions of the beta function within the cell. In this paper, we study the fast ion instability with account of the spatial inhomogeneity of ion and beam parameters.

For the sake of simplicity, we focus on the interaction of an electron beam with ions, although similar effects apply to a positron beam trapping free electrons. We also assume a one-dimensional model that treats only vertical linear oscillation of the beam and ion centroids.

As in Refs. [1, 2], we adopt a model that treats the bunch train as a continuous beam. This model is applicable if the distance between the bunches $l_{b}$ is smaller than the betatron wavelength, $l_{b} \ll c / \omega_{\beta}$, and the ion oscillation wavelength, $l_{b} \ll c / \omega_{i}$.

\section{THEORY}

We will use the following equation of motion from [2] in which we now include the effect of spatial variation of parameters along the beam path,

$$
\begin{array}{r}
\frac{\partial^{2} y(s, z)}{\partial s^{2}}+K(s) y(s, z) \\
=-\kappa(s) \int_{0}^{z} z^{\prime} \frac{\partial y\left(s, z^{\prime}\right)}{\partial z^{\prime}} D\left(s, z-z^{\prime}\right) d z^{\prime},
\end{array}
$$

where $y$ is the offset of the centroid of the beam as a function of longitudinal position $s$ and the variable $z$ measures the distance along the bunch train, $z=c t-s, K(s)$ is the focusing strength of the lattice, $D$ is the decoherence function of the ions, and $\kappa$ is the coefficient responsible for the beam-ion interaction,

$$
\kappa \equiv \frac{4 \dot{\lambda}_{i o n} r_{e}}{3 \gamma c \sigma_{y}\left(\sigma_{x}+\sigma_{y}\right)},
$$

where $\gamma$ denotes the relativistic factor for the beam, $r_{e}$ is the classical electron radius, $\sigma_{x, y}$ is the horizontal and vertical rms-beam size respectively, and $\dot{\lambda}_{i o n}$ is the number of ions per meter generated by the beam per unit time. For a given cross section $\sigma_{i}$ for collisional ionization, we have

$$
\dot{\lambda}_{\text {ion }}\left[\mathrm{m}^{-1} \mathrm{~s}^{-1}\right] \approx 0.9 \cdot 10^{9} \sigma_{i} n_{e} p_{\text {gas }},
$$

where $n_{e}$ is the number of electrons in the beam per meter, $p_{\text {gas }}$ is the residual gas pressure in torr, and $\sigma_{i}$ is the cross section in Mbarns ( $\sigma_{i}$ is about 2 Mbarns for carbon monoxide ionized by $40 \mathrm{GeV}$ electron beam). The decoherence function is determined by the following relation,

$$
D(s, z)=\int d \omega_{i} \cos \left(\omega_{i} z / c\right) f\left(s, \omega_{i}\right)
$$

where $f\left(s, \omega_{i}\right)$ is the distribution function of ions over the frequency of transverse oscillations $\omega_{i}$ normalized so that $\int f\left(s, \omega_{i}\right) d \omega_{i}=1$. The decoherence function represents the oscillation of the centroid of an ensemble of ions which is initially offset from the equilibrium position by one unit.

We will also assume that the interaction between the beam and the ions is small,

$$
c^{2} \kappa l \ll \omega_{i}^{2}, \omega_{\beta}^{2},
$$


so that the instability develops on a time scale which is much larger than both the betatron period and the period of ion oscillations. Typically this inequality is easily satisfied in the experiment. In such a situation, the most unstable solution of Eq. (1) can be represented as a betatron wave propagating in the beam with a varying amplitude,

$$
y(s, z)=\operatorname{Re} A(s, z) \sqrt{\beta(s)} e^{-i \psi(s)+i \omega_{i 0} z / c},
$$

where the complex amplitude $A(s, z)$ is a 'slow' function of its variables,

$$
\left|\frac{\partial \ln A}{\partial s}\right| \ll \frac{\omega_{\beta}}{c},\left|\frac{\partial \ln A}{\partial z}\right| \ll \frac{\omega_{i 0}}{c},
$$

$\beta(s)$ is the beta function, $\psi(s)$ is the betatron phase, and $\omega_{i 0}$ is the averaged over the ring ion frequency, $\omega_{i 0}=$ $C^{-1} \int d \omega d s \omega f(s, \omega)$, where $C$ is the circumference of the ring. For a fixed $z$ and constant $A$, the $s$-dependence in Eq. (6) describes a pure betatron oscillation, while, for a fixed $s$ (that is in the ion rest frame), the $z$-dependent part implies oscillations with the frequency $\omega_{i 0}$. Hence the wave resonantly couples the ions and the electrons.

It is reasonable to assume that, with a good accuracy, the variation of the ion frequency from point to point does not change the shape of the ion distribution function but only shifts it along the frequency axis,

$$
f\left(s, \omega_{i}\right)=F\left(\omega_{i}-\omega_{i 0}-\delta \omega_{i}(s)\right),
$$

where $\delta \omega_{i}(s)$ is the deviation of the local ion oscillation frequency in the center of the beam from $\omega_{i 0}$. We will also assume that $\delta \omega_{i}$ is small, $\delta \omega_{i}(s) \ll \omega_{i 0}$; in the opposite case, when $\delta \omega_{i}$ is comparable to $\omega_{i 0}$, the large variations of the ion frequency should strongly suppress the instability.

Substituting Eqs. (4), (6), and (8) into Eq. (1) and neglecting the second derivative $\partial^{2} A / \partial s^{2}$ on the right hand side gives the following equation

$$
\begin{aligned}
\frac{\partial A(s, z)}{\partial s} & =\frac{\kappa \omega_{i 0} \beta}{2 c\left(2+i \beta^{\prime}\right)} \int_{0}^{z} z^{\prime} d z^{\prime} A\left(s, z^{\prime}\right) e^{-i \omega_{i 0}\left(z-z^{\prime}\right)} \\
& \times \int d \omega_{i} f\left(s, \omega_{i}\right)\left[e^{-i \omega_{i}\left(z-z^{\prime}\right)}+e^{i \omega_{i}\left(z-z^{\prime}\right)}\right] .
\end{aligned}
$$

If we now assume that the length of the bunch train $l$ is much larger than the ion wavenumber, $l \omega_{i} / c \gg 1$, and recall that the ion frequency $\omega_{i}$ is close to $\omega_{i 0}$, we conclude that the first term in the brackets rapidly oscillates with the frequency $\approx 2 \omega_{i 0}$, whereas the second term is a relatively smooth function of $\left(z-z^{\prime}\right)$. Clearly, the second term makes the dominant contribution. Leaving only this term in the equation leads to the following expression for the amplitude $A$,

$$
\frac{\partial A(s, z)}{\partial s}=\frac{\kappa \omega_{i 0} \beta}{2 c\left(2+i \beta^{\prime}\right)} \int_{0}^{z} z^{\prime} A\left(s, z^{\prime}\right) \hat{D}\left(s, z-z^{\prime}\right) d z^{\prime},
$$

where

$$
\hat{D}(s, z)=e^{i \delta \omega_{i}(s) z / c} D_{0}(z),
$$

and

$$
D_{0}(z)=\int d \omega F(\omega) e^{i \omega z / c}
$$

is the decoherence function in the homogeneous case (see Ref. [2]).

It has been shown in Ref. [2] that the decoherence function $D_{0}(z)$ can be approximated by the following expression, $D_{0}(z)=\left(1+i \alpha \omega_{i 0} z / c\right)^{-1 / 2}$, where $\alpha=3 / 8$ is a numerical factor. This function decays very slowly as $z \rightarrow \infty$, which explains why the effect of decoherence (equivalent, in some sense, to Landau damping) only insignificantly decreases the growth rate of the instability [2]. Mathematically, slow decay manifests itself in the fact that $\int_{0}^{\infty} D_{0}(z) d z$ does not converge at infinity.

Situation completely changes for inhomogeneous case. Now, because of the presence of the oscillating factor in Eq. (11), the integral $\int_{0}^{\infty} \hat{D}(s, z) d z$ converges almost everywhere (except the points where $\delta \omega_{i}=0$ ), and we can introduce a (complex) decoherence length $l_{d}$ :

$$
l_{d}(s)=\int_{0}^{\infty} \hat{D}(s, z) d z .
$$

A simple picture of instability arises in the limit of short decoherence length

$$
\left|l_{d}\right| \ll l .
$$

In this limit, the main contribution to the integral on the right-hand side of Eq. (10) comes from the region where $z^{\prime} \approx z$, and we can put $z^{\prime} A\left(z^{\prime}\right)$ out of the integral substituting $z$ for $z^{\prime}$,

$$
\frac{\partial A(s, z)}{\partial s}=\Lambda(s, z) A(s, z)
$$

where

$$
\Lambda(s, z)=\frac{\kappa(s) \omega_{i 0} \beta(s) z l_{d}(s)}{2 c\left(2+i \beta^{\prime}(s)\right)} .
$$

The solution to the last equation is,

$$
A(s, z)=A_{0}(z) \exp \left[\int_{0}^{s} \Lambda\left(s^{\prime}, z\right) d s^{\prime}\right],
$$

which means that the instability develops with the averaged growth rate $\Gamma$

$$
\Gamma(z)=c\langle\operatorname{Re} \Lambda(s, z)\rangle .
$$

where the angular brackets denote averaging over a period of the lattice (or circumference in a circular accelerator). As a matter of fact, $\Gamma(z)$ is a linear function of $z$.

Equation (16) predicts how the growth rate scales with the parameters of the beam and the vacuum pressure. For large variation of the ion frequency, $\delta \omega_{i} / \omega_{i 0}>0.3$, the decoherence length $l_{d}$ is inversely proportional to the ion frequency, $l_{d} \sim c / \omega_{i} 0$, and we obtain the following rough 
estimate for the maximal growth rate at the end of the bunch train,

$$
\Gamma \sim \frac{c \kappa(s) \beta l}{4} .
$$

This equation gives a scaling of the growth rate of the instability with the bunch current $I_{b}$, number of bunches in the train $n_{b}$, and the vacuum pressure $p$,

$$
\Gamma \propto p n_{b} I_{b}=p I,
$$

where $I=n_{b} I_{b}$ is the total beam current. This scaling differs from the regime when $\left|l_{d}\right| \gg l$ studied in Refs. [1, 2] where the characteristic time $\tau$ of the instability scales as $\tau^{-1} \propto p n_{b}^{2} I_{b}^{3 / 2}$.

\section{GROWTH RATE OF THE INSTABILITY FOR THE ALS EXPERIMENT}

Using Eq. (18) we calculated the growth rate of the fast ion instability for the conditions of the dedicated experiment in the Advanced Light Source at the LBL [3]. The horizontal and vertical beta functions for one period of the ALS lattice are shown in Fig.1. We assumed the following parameters in the calculation: beam energy $1.5 \mathrm{GeV}$, accelerator circumference $196.8 \mathrm{~m}$, bunch spacing $0.61 \mathrm{~m}$, vertical emittance $9.4 \times 10^{-11} \mathrm{~m}$, horizontal emittance $4.1 \times 10^{-9} \mathrm{~m}$.

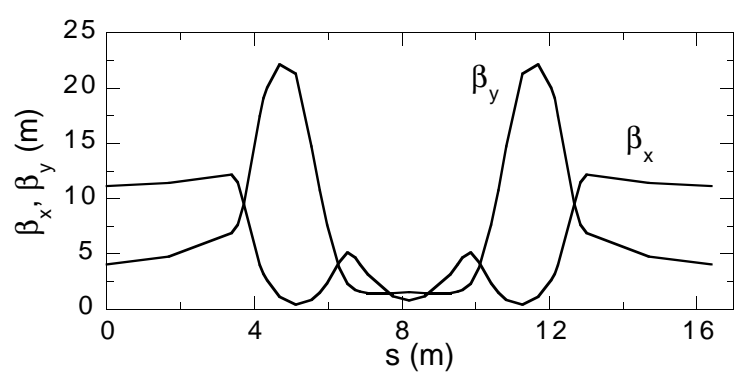

Figure 1: Plot of horizontal and vertical $\beta$-functions in the ALS. Only one period of the lattice is shown.

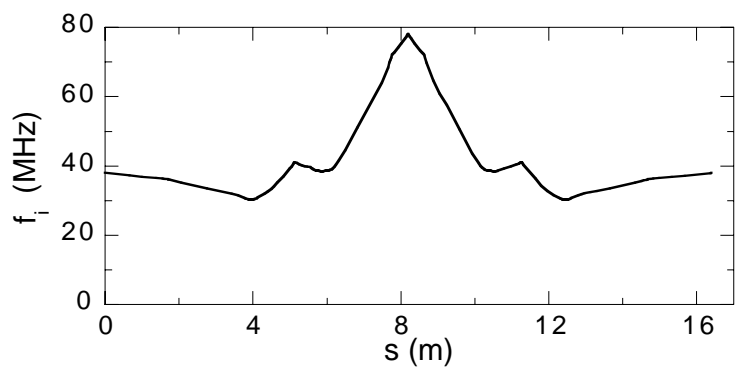

Figure 2: Frequency of $\mathrm{He}$ ion oscillations as a function of position for the lattice shown in Fig. 1. Beam current $I=0.2 \mathrm{~A}$, number of bunches $n_{b}=240$.

We also assumed a residual $\mathrm{He}$ gas of pressure $p=80$ nTorr [3] and used the ionization cross section of 0.15 Mbarn [5]. The variation of the ion frequency along the beam path in one period of the ring is shown in Fig 2, and the ratio of the calculated decoherence length given by Eq.
(13) and the bunch train length $l$ is shown in Fig. 3. One sees that the inequality (14) holds almost everywhere except for two positions in the half-period of the ring. The average ion frequency for this case is $41 \mathrm{MHz}$, with the rms spread of about $12 \mathrm{MHz}$.

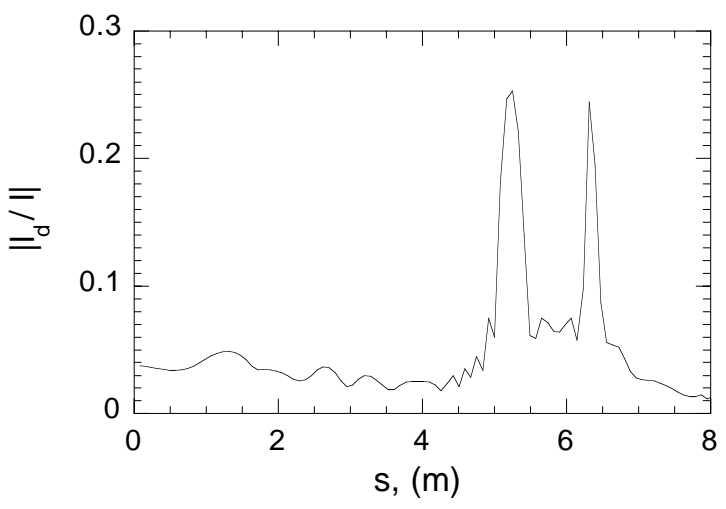

Figure 3: Absolute value of the ratio of the decoherence length and the train length as a function of position in a half of the ALS period.

Table 1: Inverse growth rate of the instability

\begin{tabular}{lll}
$I, \mathrm{~A}$ & $n_{b}$ & $\Gamma^{-1}, \mathrm{~ms}$ \\
\hline 0.2 & 240 & 0.40 \\
0.1 & 240 & 0.83 \\
0.2 & 150 & 0.34 \\
0.2 & 320 & 0.42
\end{tabular}

The results of the calculations for several different regimes are given in Table 1 . The growth times predicted by Eq. (18) turns out to be orders of magnitude larger than predicted by the theory that assumes a constant ion frequency in the ring. The growth rate for a given total current is almost independent of the train length, in accordance with the scaling law (20).

\section{REFERENCES}

[1] T. O. Raubenheimer and F. Zimmermann. Phys. Rev. E, 52, 5487, (1995).

[2] G. V. Stupakov, T. O. Raubenheimer, and F. Zimmermann. Phys. Rev. E, 52, 5499, (1995).

[3] J. Byrd et al. Paper 3B.11, these proceedings; to be published in Physical Review Letters.

[4] G.V. Stupakov. Proceedings of the International Workshop on Collective Effects and Impedance for B-Factories (CEIBA95), Tsukuba 12-17 June, 1995, KEK Proceedings 96-6, Aug. 1996, p. 243.

[5] Tables and Graphs of Electron-Interaction Cross Section from $10 \mathrm{eV}$ To $100 \mathrm{GeV}$ Derived from LLNL Evaluated Electron Data Library (EEDL), Z=1-100. DE92-007986, Lawrence Livermore National Laboratory, 1991. 